\title{
Analysis of field-grown Panax ginseng rhizosphere microbiome provides clues for preventing red skin root syndrome
}

\section{Xingbo Bian}

Jilin Agricultural University

Ling Dong

Jilin Agricultural University

\section{Yan Zhao}

Jilin Agricultural University

He Yang

Jilin Agricultural University

\section{Yonghua Xu}

Jilin Agricultural University

\section{Yongzhong Han}

Jilin Provincial Ginseng and Pilose Antler Office

Lianxue Zhang ( $\nabla 84544360 @ q q . c o m$ )

Jilin Agricultural University

\section{Research Article}

Keywords: Ginseng red skin root syndrome, rhizosphere, soil ecological environment, microbial interaction network

Posted Date: January 21st, 2021

DOI: https://doi.org/10.21203/rs.3.rs-148957/v1

License: (c) (1) This work is licensed under a Creative Commons Attribution 4.0 International License. Read Full License 


\section{Abstract \\ Background}

Ginseng red skin root syndrome (GRS) is one of the most common ginseng diseases. It leads to a severe decline in ginseng quality and seriously affects the ginseng industry in China. However, as a root disease, the characteristics of GRS rhizosphere microbiome are still unclear.

\section{Methods}

The amplicon sequencing technology, combined with bioinformatics analysis, was used to explore the relationship between soil ecological environment and GRS.

\section{Results}

There were significant differences in the diversity and richness of soil microorganisms between the rhizosphere with different degrees of disease, especially between healthy ginseng (HG) and heavily diseased groups. We also found that bacterial communities underwent multiple changes between complex stability and simple instability in different ginseng rhizospheres through the established interaction networks. The GRS group also had more competition with each other and ecological niche separation than the HG group. The fungal community's stability decreased significantly in the early stages of the disease, followed by the formation of a stable and complex fungal community. The GRS groups significantly increased interspecies cooperation and ecological niche overlap in the fungal network than the HG group. Microbes closely related to potential pathogenic fungi were also identified according to the interaction network, which provided clues for looking for biological control agents. Finally, the Distance-based redundancy analysis (dbRDA) results indicated that total P (TP), available K (AK), available $P(A P)$, catalase (CAT), invertase (INV) are the key factors that influence the microbial communities.

\section{Conclusions}

This study collectively analyzed the changing characteristics in ginseng rhizosphere and provided the basis for soil improvement and biological control of field-grown ginseng.

\section{Introduction}

Ginseng (Panax ginseng Mayer), mainly distributed in northeast China and South Korea, is known as the "King of Herbs" because of its substantial medicinal value. Because of the continuous cropping effect of ginseng, the method of cutting and planting ginseng was often used in China before. For a long time, we have lost a lot of forest resources for the ginseng industry. And now, this type of cultivation is no longer 
allowed. Due to the limitations of planting areas and planting patterns, it is more important to improve the quality and yield of ginseng for the sustainable development of the ginseng industry. Many diseases that occur during the growth process of ginseng are the biggest obstacles to our obtaining high yield and quality ginseng.

GRS has been reported as a disease caused by $\mathrm{Al}, \mathrm{Fe}$, and microbial stress in ginseng cultivation. The condition is characterized by rust-stained reddish-brown areas on the epidermis of ginseng roots. Areas may grow more significant as the planting years increase. In severe cases, areas can occupy more than $80 \%$ of the epidermis of ginseng roots. Unlike root rot disease, severe symptoms of red skin roots are also rarely associated with root rot. In the stem and leaf part of ginseng, however, there is no prominent characteristic. Previous studies have found that the application of chitosan induces red skin roots, and a variety of phenolic compounds and elements accumulate in red skin tissues [1]. In particular, the accumulation of $\mathrm{Al}$ and Fe may promote the accumulation of phenolic compounds and the activation of enzymes related to their oxidation. The activity of various antioxidant substances and antioxidant enzymes in red skin tissues is significantly increased, which can prevent phenolic compounds from being oxidized [2]. The microorganisms produce pectinase, cellulase and ligninase that damage the cell walls of ginseng roots, causing red skin symptoms that are exacerbated by the application of $\mathrm{Fe}^{3+}$ [3]. The presence of pathogenic fungi has been reported, and non-biological factors in the red skin formation process seem to be closely related [4-9].

There are close interactions between plants, soil and microorganisms[10]. Healthy plant growth is dependent on the ability of microorganisms to promote soil material circulation and nutrient conversion $[11,12]$. The plants' root diseases are closely related to soil microbial community and physical and chemical properties, and there are a variety of potential pathogens in soil [13]. Therefore, to study a plant root disease, it is necessary to understand the microbial community and physical and chemical properties of rhizosphere.

The results of previous studies showed that the rhizosphere of diseased ginseng was separated from that of healthy ginseng, and there are considerable differences in the dominant bacteria and fungi genera in the two rhizospheres. Some beneficial microorganisms were significantly reduced in the rhizosphere of diseased ginseng, and the potential pathogen Ilyonectria was also identified [6]. And the diversity of bacteria and fungi in the rhizosphere of diseased ginseng was decreased [14]. There have also been studies exploring rhizospheres of plants with varying degrees of red skin and have found a strong correlation between metal element accumulation and red skin symptoms. According to the experimental results, llyonectria may not be necessary for red skin root [15].

In this study, we classified the ginseng rhizosphere into five degrees based on the severity of the disease. The 16S rRNA gene and ITS region were applied to explore the five degrees rhizosphere microbial community composition on the Illumina PE250 platform. Nutrient composition and enzyme activity in rhizosphere were also examined, and their relationship with microbial changes was explored. This study 
elucidated the evolution rule of ginseng rhizosphere with the aggravation of GRS and provides the basis for preventing and treating this disease in field production.

\section{Results}

\section{richness and diversity of microbial communities}

In the bacterial analysis based on 16S rRNA, the OTUs number in the GRS2 group was significantly higher than that in the HG group, GRS1 group, and GRS3 group $(P<0.05)$. The Observed species of the GRS2 group is also substantially higher than HG and GRS3 $(P<0.05)$. Besides, The Shannon index and Simpson index of the GRS2 group are both significantly higher than that of other groups, and the Chao 1 index of GRS2 group is also significantly higher than that of the HG group and GRS3 group $(P<0.05)($ Table 1$)$.

Based on the ITS rRNA analysis of fungi, there was no significant difference in OTUs numbers among the five groups. The Observed species and Chao 1 index of the GRS2 group were significantly higher than that of the GRS3 group $(P<0.05)$. Also, the Shannon indices of the $H G, G R S 1$, and GRS2 groups were considerably higher than those of the GRS3 and GRS4 groups $(P<0.05)$. The Simpson index was significantly higher in the HG group than in the GRS3 and GRS4 groups $(P<0.05)$ (Table 1).

\section{Differences and composition of microbial communities}

NMDS analysis based on bacterial OTUs level showed no significant separation of GRS2, GRS3, and GRS4 groups in all five groups of samples, while HG and GRS1 groups were separated from other groups (Fig. 1A). Cluster analysis showed that samples from the HG and GRS3 groups were significantly clustered to form independent clusters. The samples were then analysed for bacterial abundance, belonging to 46 phyla, 246 families, and 593 genera. In the analysis of phylum level, Proteobacteria is the most dominant phylum (average 50.2\%), followed by Acidobacteria (average 17.6\%), Bacteroidetes (average 7.1\%) Gemmatimonadetes (average 6.0\%), and Actinobacteria (average 5.9\%) (Fig. 2A).

In terms of the fungi community, by NMDS analysis, we found the significant separation of HG and GRS2 groups from GRS3 and GRS4 groups (Fig. 1B). UPGMA cluster analysis showed that HG, GRS3, and GRS4 samples were significantly clustered and formed independent clusters. Furthermore, based on the ITS gene sequences annotation, we identified 12 phyla, 197 families, and 323 genera in the fungal community. At the phylum level, Ascomycota is the most dominant (average 53.8\%), followed by Mortierellomycota (average 16.0\%), Basidiomycota (average 7.6\%), and Glomeromycota (average 1.3\%) (Fig. 2B).

\section{Biomarkers and different levels of taxa change}

Through LEfSe analysis, we found six bacterial taxa in the HG group with LDA scores greater than 4, including Acidobacteria and Actinobacteria. In the GRS1 group, LDA scores greater than 4 were Alphaproteobacteria and Rhizobiales. Phyla level with LDA scores greater than 4 in GRS3 was Proteobacteria and Bacteroidetes. Potential biomarkers of the GRS4 group were selected as Rizobiaceae 
and Achromobacter (Fig. 3A and Fig. 3B). Then, we conducted MetaStat analysis (from the phylum level to the species level) to understand further the bacterial community changes with the disease severity of ginseng (Fig. S2). The results showed that the bacterial abundance in rhizosphere at different levels varied significantly with varying degrees of disease. Notably, at the phylum level, compared with the HG group, the abundance of Proteobacteria in the four red skin groups was significantly increased, and Bacteroidetes has a greater abundance in GRS3 and GRS4 groups. Besides, the abundance of Actinobacteria, Chloroflexi, and Gemmatimonadetes were lower in the severe groups (GRS3 and GRS4) than in the healthy and mild groups (GRS1 and GRS2). Interestingly, the genera with a relatively high abundance were concentrated in HG, GRS3, and GRS4 group, and there were also significant differences between the two severe groups (GRS3 and GRS4).

LEfSe analysis results for fungi showed that, in the HG group, Fusariun, Chaetomium, and Glomeromycota had LDA scores over 4. In the GRS1 and GRS2 group, Mortierellomycota and Humicola were screened out, respectively. Besides, there were more taxa with LDA scores above 4 in the GRS3 group, and they were concentrated in Ascomycota, including Nectriaceae, Herpotrichiellaceae, and Ilyonectria (Fig. 3C and Fig. 3D). For the MetaStat analysis at the phylum level, the results showed that the relative abundance of Mortierellomycota, Glomeromycota, and Mucoromycota decreased with increasing ginseng red skin area (Fig S3). Besides, among several fungi with greater relative abundance at the genus level, the GRS3 and GRS4 groups had a much greater abundance of Ilyonectria than the other three groups. In contrast, the abundance of Trichosporon and Paraglomus decreased with the increase of red skin area. The Humicola abundance of GRS2 and GRS3 groups was higher than the other three groups (Fig. S3).

\section{Network analysis of microbial communities}

Based on spearman correlation, microbial networks in five groups from HG to GRS4 were established. Through microbial networks, we can find that the keystone phyla of bacteria and fungi are Proteobacteria and Ascomycota, respectively (Fig. 4 and Fig. 5). In the bacterial network, the number of nodes in the five groups did not change significantly. However, the number of total links in the five groups was not stable, reaching the maximum in the GRS4 group (3600). All four red skin groups increased their proportion of negative links compared to the HG group (Table S3). Among other topological properties, the average path length of the GRS4 group was lower than that of other groups. The modularity of red skin groups was more down than the HG group, especially the GRS4 group. Also, the GRS4 group clustering coefficient and average degree were higher than the other groups (Table S3).

For fungi, the GRS4 group had significantly more links than the other groups, and the four red skin groups had more proportion of negative links than the HG group (Table S3). Also, from HG to the GRS4 group, the modularity value decreases gradually. The average path length in the GRS4 group also decreased significantly. In contrast, the clustering coefficient and average degree increased for the GRS4 group (Table S3). 
In addition, through the interaction network, we obtained the microbe that may be strongly correlated with GRS-related fungus (Ilyonectria, Cylindrocarpon, and Fusarium) mentioned in the previous studies (Table S4) [8] [5] [16, 17]. The results showed that the network relationship of the three fungi was different in the rhizosphere of ginseng with different degree of disease, and the interaction relationship may be more in GRS4 group.

\section{Soil properties and their relationship with microbial communities}

After determining soil physical and chemical properties, we found that the $\mathrm{pH}$ of the two severe groups (GRS3 and GRS4) decreased significantly compared with the other three groups (Fig. S4). Significant changes in some nutrients were also observed among the sample groups (Fig. S5). The levels of AP and TP were lower in the two severe groups than in the HG group and mild groups (GRS1 and GRS2), and the AK content of GRS1, GRS3, and GRS4 groups was significantly lower than that of HG and GRS2 groups (Fig. S5D-E and Fig. S5G). Besides, there were no significant changes in other nutrients (OM, TN, AN, and TK). For rhizosphere enzyme activities, the CAT and PHO enzymes in the GRS3 and GRS4 groups were significantly lower than those in the other three groups (Fig. S6A and Fig. S6D), and the INV in the GRS4 group was also significantly reduced (Fig. S6B).

The dbRDA results for fungi and bacteria demonstrate the relationship between microorganisms and environmental factors. In Fig. 6A, axis 1, and axis 2 accounts for $75.81 \%$ and $11.51 \%$ of the total variance. TP, AK, CAT, AP, and $\mathrm{pH}$ were significantly correlated with bacterial phylum composition. In the fungi plot, axis 1 and axis 2 account for $71.55 \%$ and $14.71 \%$ respectively, and INV, CAT, and AP are most closely related to the community composition of fungi(Fig. 6B).

\section{Discussion}

GRS is one of the most concerned ginseng diseases at present. In recent years, GRS has appeared in large areas in some ginseng producing areas in China, seriously damaging the ginseng industry. As a root disease, a thorough understanding of its rhizosphere characteristics is essential [18]. In this study, to provide basis for soil improvement and disease control, we made a more detailed division of the disease (from HG to GRS4) to comprehensively reveal the pattern of rhizosphere changes in this ginseng disease's background.

\section{Changes of microbial diversity and richness in rhizosphere}

Through the statistics of the Shannon index and Simpson index of each group, we found that with the increase of ginseng disease degree, the change of bacterial community diversity in rhizosphere was firstly increased and then decreased. Observed species and Chao1 index indicated that the richness of the rhizosphere bacterial community fluctuated in these five degrees, and the highest richness was found in the GRS2 group (Table 1). This result seems to imply that GRS2 (red skin area 25 to $50 \%$ ) is a grade of concern because of the turning point of changes in the ginseng rhizosphere's bacterial diversity. 
For fungi, the fungal diversity in the severe groups (GRS3 and GRS4) was significantly lower than that in the HG group, while the richness did not change significantly (Table 1). Overall, the fungal diversity in the GRS's rhizosphere was affected, especially in severe groups.

\section{Changes of microbial community composition in rhizosphere}

To better reflect the nonlinear structure of the ecological data and the inter- and intra-group differences in the samples, we performed NMDS analysis based on OTUs level (Fig. 1). The bacteria in HG and GRS1 groups were separated from the other three groups, which means that with the development of the disease, the bacteria in rhizosphere had a continuous change process and tended to be stable at the later stages (from GRS2 to GRS4) of development (Fig. 1A). For the NMDS analysis of fungi, we still found that the HG group was significantly separated from the GRS3 group and the GRS4 group. Interestingly, the groups were not separated by the degree of disease development (Fig. 1B). The possible reason is that the fungal community develop fluctuating rather than gradual as the disease progresses. Further, by clustering analysis, we also found that the bacterial HG group was individually clustered; meanwhile, the other GRS groups were not incredibly significantly clustered, confirming the bacterial community's instability in the rhizosphere of the diseased ginseng (Fig. 2A). In terms of fungi, in addition to the HG group, the GRS3 group and GRS4 group also had obvious clustering, which seemed to indicate that the fungal microbial community in the severe groups again formed the stable state different from the HG group. (Fig. 2B). Based on the above, the bacterial community of ginseng rhizosphere appears to be more sensitive than the fungal community at the beginning of the disease. On the contrary, the fungal community has more stability compared to the bacterial community.

\section{Characteristics of microbial community taxa variation in rhizosphere}

Proteobacteria is the most dominant phylum among bacteria, and its abundance increases with the degree of disease (Fig. 2A and Fig. S2). The increase of Bacteroidetes abundance in GRS3 and GRS4 groups (Fig. S2) may be due to its good degradation ability, which is more suitable for survival in the fiercely competitive soil[19]. The abundance of Actinobacteria, Chloroflexi, and Gemmatimonadetes was reduced in severe groups (GRS3 and GRS4) (Fig. S2). Actinobacteria contribute to OM's the decomposition [20], but the ecological functions of Chloroflexi and Gemmatimonadetes were unclear. In the LEfSe analysis, 24 biomarkers were obtained and distributed in four groups (Fig. 3A). We also identified Acidobacteria as a potential biomarker for the HG group, which has been reported to degrade plant-derived OM specifically and is more abundant in soils of enriched plants [21]. In the GRS1 group, Rhizobiales, a clade of Alphaproteobacteria, were screened out and reported to fix nitrogen or as a pathogen[22]. Most biomarkers in the GRS3 group belongs to Proteobacteria, and the other belong to Bacteroidetes. Although described as phytopathogenic, the GRS4 group biomarker, Rhizobiaceae, is not known whether it is related to rust roots [23].

The fungal community's dominant phyla are Ascomycota, Mortierellomycota, and Basidiomycota (Fig. 2B). In severe groups, the dominance of Mortierellomycota decreased (Fig. S3). Besides, there are Glomeromycota and Mucoromycota that decrease in abundance as the disease progresses. In the 
present study, we still found changes in the abundance of Ilyonectria in different groups at the genus level. We also found the fungi genus (Trichosporon and Paraglomus), whose abundance decreases with disease development (Fig. S3). We also obtained specific biomarkers for each group through LEfSe analysis, such as Glomeromycota, Fusarium, and Chaetomium of the HG group (Fig. 3C). In the GRS3 group, biomarkers all belong to Ascomycota, the soil ecosystem's main fungal decomposer (Fig. 3D) [24, 25]. Among them, except Ilyonectria, the other biomarkers were not associated with GRS, although they have been reported to be associated with plant diseases [26, 27].

In general, we used statistical methods to look for changes in the composition of rhizosphere microbial communities with different disease degrees. Although taxa were found to be correlated (positively or negatively) with disease severity, their ecological function was unknown.

\section{Changes of the microbial interactions in the networks}

Co-occurrence networks can be constructed to analyze the interaction and co-existence patterns among different microorganisms, which is crucial to our further understanding of the changes in rhizosphere with GRS development [28].

Fig. 4 shows that the phylum with the highest connectivity in the five groups' bacterial community is Proteobacteria, which is consistent with previous study[29]. Therefore, with the disease's development, the keystone phylum has not changed in ginseng rhizosphere. However, it should be noted that Proteobacteria accounts for a larger proportion in GRS3 and GRS4 group. Since Proteobacteria could exploit labile carbon sources and produce extracellular polysaccharides to bond sand particles, this may mean that the rhizosphere of the severe groups is more solid, while has a stronger nutrient metabolism $[30,31]$. Further, from the obtained topology parameters, we found that links and average degree were fluctuating (from HG to GRS4). The average path length of the GRS4 group was significantly lower than the other four groups (Table S3). Thus, with the disease's development, the bacterial community in the ginseng rhizosphere underwent multiple changes from complex and stable to simple and unstable. In addition, by counting the ratio of positive to negative links, we can also predict that there may be more interspecific competition and ecological niche separation in the GRS groups than in the HG group (Table S3) [32].

For the fungal networks, we can find that the keystone phylum of rhizosphere was Ascomycota, unchanged at all disease development stages. It can be seen that in ginseng rhizosphere, Ascomycota was essential for resisting the harsh environment and maintaining system stability [33]. Through the statistics of topology parameters, we found that from HG to GRS4, the number of links, clustering coefficient, and the average degree were the lowest in the GRS1 group, and then gradually increased (Table S3). Therefore, in the early stage (GRS1) of ginseng disease, fungal communities' interaction and stability decreased. Interestingly, after this, the soil's fungi gradually formed a complex and stable community (GRS4). From the change in the ratio of positively and negatively links, it appears that the competitive relationships and the taxa in the same ecological niche were severely affected in the GRS1 group (Table S3). Furthermore, the negative correlation in GRS groups was lower than that in the HG 
group. In previous studies, GRS was closely related to fungi [8] [34]. Here, based on the interaction network, we identified microorganisms that interacted closely with the reported potentially pathogenic fungi. Of these, Simplicillium, which is extremely negatively correlated with llyonectria, is reported as a biological control agent [35]. In addition, although these microorganisms, such as Musicillium and Arachnotheca, have not been fully studied, the establishment of the networks also provides a basis for the future search for microorganisms that have antagonistic interactions with pathogens and, thus, for biocontrol development.

\section{Changes of physicochemical properties and their relationship to microbial communities}

Changes in the soil's physicochemical properties directly determine the structural composition of the microbial community $[36,37]$. In this study, $\mathrm{pH}$, nutrient composition, and enzyme activity of rhizosphere were measured from HG to GRS4 group. From the GRS2 group, the $\mathrm{pH}$ of rhizosphere decreased significantly (Fig. S4). Compared with other groups, AP and TP in rhizosphere of the severe groups (GRS3 and GRS4) were reduced considerably. Also, AK's content was different among the groups, while there was no significant difference in other nutrients (Fig. S5). In addition, the activities of three enzymes (CAT, INV, and PHO) were significantly decreased in the rhizosphere of the severe groups (GRS3 and/or GRS4), while the activity of the URE was not significantly changed (Fig. S6). The results indicated that the development of GRS was correlated with the physicochemical properties of rhizosphere. The reasons for the above changes in physicochemical properties may be related to the occurrence of diseases affects the production of enzymes and the conversion of nutrients in the soil or increases their consumption[38, 39]. It is also associated with the release and accumulation of secretions from the root of diseased ginseng [2].

To explore the microbial-environmental linkages and gain insight into the key factors influencing microbial communities' changes, we used dbRDA to examine microbial communities and environmental factors $[40,41]$. In the dbRDA results for bacteria, TP, AK, CAT, and AP were the main factors determining the distribution of bacterial community composition and taxa changes (Fig. 6A). In the dbRDA analysis of fungi, INV, CAT, and AP were significant factors affecting the structure change of the fungal community (Fig. 6B). This study suggests that AP and CAT may focus on future research on soil improvement due to their close correlation with both bacterial and fungal communities.

\section{Conclusions}

we studied the changes of soil ecological environment of ginseng rhizosphere with different red skin degree in a same ginseng farm. With the development of the red skin degree, the bacterial community's diversity first increased and then decreased, and the richness also fluctuated. The fungal community's diversity decreased obviously during the severe disease, but the richness was not affected. The rhizosphere of ginseng at different stages showed obvious differences, especially between HG and severe groups (GRS3 and GRS4). Besides, we also explored the changes of microbial taxa in different classification levels from HG to GRS4, as well as potential biomarkers for different groups. We 
established the interaction networks of rhizosphere microorganisms under this disease's background, and the keystone phylum of different groups of bacteria and fungi remained unchanged, namely Proteobacteria and Ascomycota. As the disease progressed, the bacterial community underwent multiple changes from complex and stable to simple and unstable, and the GRS group also had more intercompetition and ecological niche segregation than the HG group. The fungal community's stability decreased significantly in the early stages of the disease, followed by forming a stable and complex fungal community. In contrast to bacteria, the GRS groups significantly increased interspecies cooperation and ecological niche overlap in the fungal network than the HG group. In the network, we also obtained several microorganisms that are closely related to the potential pathogenic fungi, which can provide the basis for biological control. Soil TP, AK, AP, CAT, INV are the key factors influencing the microbial communities. Overall, our study comprehensively analyzed the variation characteristics of the ginseng rhizosphere, providing evidence for disease control and soil improvement.

\section{Materials And Methods}

\section{Site description and sample collection}

We found that the ginseng grown at a ginseng farm showed more red skin root in Hunchun city (42.86' $\mathrm{N}$ and $130.37^{\prime} \mathrm{E}$ ) in northeast China after previous investigations [42]. This area has a temperate oceanic monsoon climate. The ginseng farm had an average annual rainfall of $757 \mathrm{~mm}$ and an average yearly temperature of $3.5^{\circ} \mathrm{C}$ during ginseng cultivation (2015 to 2019). And this farm is the first to grow ginseng, the application of pesticides strictly comply with the"Ginseng safe production technical specification of pesticide application (DB22/T 1233-2019)".

The sampling date is July, 2020. All soil samples were collected from the above ginseng farm with ginseng (5-year-old). The rhizosphere was divided into five grades according to the degree of disease of ginseng, namely healthy ginseng (HG), rust root area 0 to $25 \%$ (GRS1), rust root area 25 to $50 \%$ (GRS2), rust root area 50 to $75 \%$ (GRS3) and rust root area greater than 75\% (GRS4). Six rhizosphere samples were collected at each grade, a total of 30 samples. The rhizosphere was collected according to Riley and Barber's standards $[43,44]$. In brief, dig out the complete ginseng roots from the soil, gently shake off the large blocks, and then obtain the ginseng root's rhizosphere. Each soil sample was sieved through a 2 $\mathrm{mm}$ plastic mesh. Then, one part of each sample was stored at $-80^{\circ} \mathrm{C}$ for DNA extraction, and the other part was air-dried at ambient temperature for determination of $\mathrm{pH}$, enzyme activity, and soil nutrients.

\section{DNA extraction, PCR amplification, and Illumina NovaSeq sequencing}

Total genome DNA from samples was extracted using the CTAB method $[45,46]$. DNA concentration and purity were monitored on $1 \%$ agarose gels. According to the concentration, DNA was diluted to $1 \mathrm{ng} / \mu \mathrm{L}$ using sterile water.

The amplicon generation refers to the experimental method of Wang et al [47]. 16S rRNA genes of V3-V4 regions and ITS genes of ITS1-1F regions were amplified used specific primer $341 \mathrm{~F}\left(5^{\prime}\right.$ - 
CCTACGGGNGGCWGCAG-3')-806R (5'-GGACTACHVGGGTWTCTAAT-3') and ITS1F (5'-

CTTGGTCATTTAGAGGAAGTAA-3')-ITS1R(5'-GCTGCGTTCTTCATCGATGC-3') with the barcode, respectively. All PCR reactions were carried out with $15 \mu \mathrm{L}$ of Phusion® High-Fidelity PCR Master Mix (New England Biolabs), $2 \mu \mathrm{M}$ of forward and reverse primers, and about $10 \mathrm{ng}$ template DNA. Thermal cycling consisted of initial denaturation at $98^{\circ} \mathrm{C}$ for $1 \mathrm{~min}$, followed by 30 cycles of denaturation at $98^{\circ} \mathrm{C}$ for $10 \mathrm{~s}$, annealing at $50^{\circ} \mathrm{C}$ for $30 \mathrm{~s}$, and elongation at $72^{\circ} \mathrm{C}$ for $30 \mathrm{~s}$. Finally, the temperature was maintained at $72^{\circ} \mathrm{C}$ for $5 \mathrm{~min}$.

Mix the same volume of $1 \mathrm{X}$ loading buffer (contained SYB green) with PCR products and operate electrophoresis on $2 \%$ agarose gel for detection. PCR products were mixed in equidensity ratios. Then, the mixture of PCR products was purified with the Qiagen Gel Extraction Kit (Qiagen, Germany).

Sequencing libraries were generated using TruSeq® DNA PCR-Free Sample Preparation Kit (IIlumina, USA) following the manufacturer's recommendations, and index codes were added. The library quality was assessed on the Qubit@2.0 Fluorometer (Thermo Scientific) and Agilent Bioanalyzer 2100 system. At last, the library was sequenced on an Illumina NovaSeq6000 platform, and 250 bp paired-end reads were generated at Novogene Bioinformatics Technology Co., Ltd. (Beijing, China).

\section{Data analysis}

Paired-end reads were assigned to samples based on their unique barcode and truncated by cutting off the barcode and primer sequence. Paired-end reads were merged using FLASH (V1.2.7, http://ccb.jhu.edu/software/FLASH/), a high-speed and accurate analysis tool, which was designed to merge paired-end reads when at least some of the reads overlap the read generated from the opposite end of the same DNA fragment, and the splicing sequences were called raw tags [48]. Quality filtering on the raw tags was performed under specific filtering conditions to obtain the high-quality clean tags according to the QIIME (V1.9.1, http://qiime.org/scripts/split_libraries_fastq.html) quality-controlled process $[49,50]$. The tags were compared with the reference database (Silva database, using UCHIME algorithm (UCHIME Algorithm, http://www.drive5.com/usearch/manual/uchime_algo.html) to detect chimera sequences, and then the chimera sequences were removed [51,52]. Then the effective tags finally obtained. Detailed quality control information is shown in Table S1 and Table S2.

Sequences analysis was performed by Uparse software (Uparse v7.0.1001, http://drive5.com/uparse/) [53]. Sequences with $\leq 97 \%$ similarity were assigned to the same operational taxonomic units (OTUs). Furthermore, all samples' rarefaction curves also indicate the reliability of the sampling depth (Fig. S1). The representative sequence for each OTU was screened for further annotation. For each representative sequence, the Silva Database (http://www.arb-silva.de/) was used based on the Mothur algorithm to annotate taxonomic information [54]. To study the phylogenetic relationship of different OTUs, and the difference of the dominant species in different samples (groups), multiple sequence alignment was conducted using the MUSCLE software (Version 3.8.31, http://www.drive5.com/muscle/) [55]. OTUs abundance information was normalized using a standard of sequence number corresponding to the 
sample with the least sequences. Subsequent analysis of alpha diversity and beta diversity were all performed basing on this output normalized data.

\section{Rhizosphere $\mathrm{pH}$, nutrients, and enzyme activity}

The rhizosphere's $\mathrm{pH}$ value was measured with a $\mathrm{pH}$ meter/potentiometer under the soil: water ratio of 1:2.5. The soil organic matter $(\mathrm{OM})$ content was measured by the potassium dichromate external heating method. The total N (TN) was determined by the Kjeldahl method; the TP was determined by the alkali fusion molybdenum antimony colorimetry (China HJ 632-2011); the total K (TK) was determined by the sodium hydroxide fusion flame photometric method. The AP was determined by $\mathrm{NaHCO}_{3}$ extraction molybdenum-antimony colorimetry; the available N (AN) was determined by the alkali diffusion method; AK was determined with the ammonium acetate extraction fame photometric method [56].

The activities of CAT, invertase (INV), urease (URE), and phosphatase (PHO) in the soil of ginseng were measured. The activity of CAT was determined by $\mathrm{KMnO}_{4}$ titration; soil INV activity was determined by 3,5-dinitrosalicylic acid colorimetry; URE activity was determined by indophenol blue colorimetry; PHO activity was determined by disodium phenyl phosphate colorimetry method.

\section{Statistical analysis}

Alpha diversity was used to analyze the microbial community diversity in the sample and assess the microbial community's species richness and diversity differences. Five indices were selected to identify microbial alpha diversity: the observed OTUs number, the observed species number, the Chao1 index, Simpson index, and the Shannon index [47]. Beta diversity was used for comparative analysis of microbial community composition in different samples. Cluster analysis was preceded by Non-Metric Multi-Dimensional Scaling (NMDS) analysis was performed to reflect inter-group and intra-group relationships. Unweighted Pair-group Method with Arithmetic Means (UPGMA) Clustering was performed as a hierarchical clustering method to interpret the distance matrix. The Wilcoxon rank-sum test was used to analyze differences in alpha diversity and beta diversity among multiple groups. All of the indices in our samples were calculated with QIIME and displayed with R software.

Metastat analysis was used to carry out permutation test among groups to study the taxa with significant differences among groups in $R$ software, and False Discovery Rate (FDR)-corrected $P$ value ( $Q$ value) $<0.05$ was considered statistically significant. Besides, to find statistically different biomarkers, the linear discriminant analysis (LDA) effect size (LEfSe) analysis was used to identify the differentially abundant taxa. LDA score greater than 4 was used as a potential biomarker.

Spearman correlation analysis was used to study the mutual change relationship between environmental factors and microbial taxa, and $\mathrm{P}<0.05$ was considered a significant difference [57]. Furthermore, dbRDA analysis was used to reflect the relationship between community structures and environmental factors [58]. Both analyses were performed using the vegan package in $\mathrm{R}$ software. 
To explore the microbial taxa with close interaction, we calculated the Spearman correlation coefficient for all samples to obtain the taxa correlation coefficient matrix. Then, the co-occurrence network was obtained through filtering. The filtering conditions were as follows: (1) remove connections with the correlation coefficient less than 0.6 , (2) filter out self-connection of nodes, and (3) remove connections with node abundance less than $0.005 \%$.

Environmental factor data were analyzed using the SPSS software (IBM Corporation, Armonk, NY, USA), and the results were expressed as the arithmetic mean value \pm standard deviation. The differences in the means were compared by the Tukey test at $\mathrm{P}<0.05$.

\section{Declarations}

Ethics approval and consent to participate: Not applicable.

Consent for publication: Not applicable.

Availability of data and material: Not applicable.

Competing interests: The authors declare that they have no competing interests.

Funding: This study was supported in part by grants from the National key R\&D program (grant No. 2017YFC1702100) and the Jilin Province Major Science and Technology Special Project (grant No. 20200504003YY).

Authors' contributions: L.Z. designed the experiment; X.B., L.D. and Y.X. analyzed the samples; Y.Z., H.Y. and Y.H. analyzed the data; X.B. wrote the first draft of the manuscript. All authors reviewed the manuscript.

Acknowledgments: The authors are grateful for the National key R\&D program (grant No. 2017YFC1702100), and the Jilin Province Major Science and Technology Special Project (grant No. 20200504003YY).

\section{References}

1. Rahman M, Punja ZK. Biochemistry of ginseng root tissues affected by rusty root symptoms. Plant Physiology Biochemistry. 2005;43(12):1103-14.

2. Zhou Y, Yang Z, Gao L, Liu W, Liu R, Zhao J, et al. Changes in element accumulation, phenolic metabolism, and antioxidative enzyme activities in the red-skin roots of Panax ginseng. Journal of Ginseng Research. 2017;41(3):307-15.

3. Lee C, Kim KY, Lee J-E, Kim S, Ryu D, Choi J-E, et al. Enzymes hydrolyzing structural components and ferrous ion cause rusty-root symptom on ginseng (Panax ginseng). Journal of microbiology biotechnology. 2011;21(2):192-6. 
4. Lu X, Jiao X, Chen A, Luo Y, Gao W. First report of Ilyonectria robusta causing rusty root of Asian ginseng in China. Plant Disease. 2015;99(1):156-.

5. Farh ME-A, Kim Y-J, Kim Y-J, Yang D-C. Cylindrocarpon destructans/llyonectria radicicola-species complex: Causative agent of ginseng root-rot disease and rusty symptoms. Journal of ginseng research. 2018;42(1):9-15.

6. Ma H, Liu D, Sun H. Deciphering microbiome related to rusty roots of Panax ginseng and evaluation of antagonists against pathogenic Ilyonectria. Frontiers in microbiology. 2019;10:1350.

7. Reeleder R, Hoke S, Zhang Y. Rusted root of ginseng (Panax quinquefolius) is caused by a species of Rhexocercosporidium. Phytopathology. 2006;96(11):1243-54.

8. Lu XH, Zhang XM, Jiao XL, Hao JJ, Zhang XS, Luo Y, et al. Taxonomy of fungal complex causing redskin root of Panax ginseng in China. Journal of ginseng research. 2020;44(3):506-18.

9. Wang Q, Xu C, Sun H, Ma L, Li L, Zhang D, et al.: Analysis of the relationship between rusty root incidences and soil properties in Panax ginseng. In: Proceedings of IOP Conference Series: Earth and Environmental Science; IOP Publishing: Bristol, UK. vol. 41; 2016: 012001.

10. Schweitzer JA, Bailey JK, Fischer DG, LeRoy CJ, Lonsdorf EV, Whitham TG, et al. Plant-soilmicroorganism interactions: heritable relationship between plant genotype and associated soil microorganisms. Ecology. 2008;89(3):773-81.

11. Gouda S, Kerry RG, Das G, Paramithiotis S, Patra JK. Revitalization of plant growth promoting rhizobacteria for sustainable development in agriculture. Microbiological Research. 2018;206:131.

12. Hou X-I, Han H, Tigabu M, Li Q-y, Li Z-x, Zhu C-I, et al. Lead contamination alters enzyme activities and microbial composition in the rhizosphere soil of the hyperaccumulator Pogonatherum crinitum. Ecotoxicology and Environmental Safety. 2021;207:111308; doi:

https://doi.org/10.1016/j.ecoenv.2020.111308.

13. Wei Z, Gu Y, Friman V-P, Kowalchuk GA, Xu Y, Shen Q, et al. Initial soil microbiome composition and functioning predetermine future plant health. Science advances. 2019;5(9):eaaw0759.

14. Wei X, Wang X, Cao P, Gao Z, Chen AJ, Han J. Microbial community changes in the rhizosphere soil of healthy and rusty Panax ginseng and discovery of pivotal fungal genera associated with rusty roots. BioMed Research International. 2020;2020.

15. Wang Q, Sun H, Xu C, Ma L, Li M, Shao C, et al. Analysis of rhizosphere bacterial and fungal communities associated with rusty root disease of Panax ginseng. Applied Soil Ecology. 2019;138:245-52.

16. Reeleder R, Brammall R. Pathogenicity of Pythium species, Cylindrocarpon destructans, and Rhizoctonia solani to ginseng seedlings in Ontario. Canadian journal of plant pathology. 1994;16(4):311-6.

17. Goswami RS, Punja ZK. Molecular and biochemical characterization of defense responses in ginseng (Panax quinquefolius) roots challenged with Fusarium equiseti. Physiological and Molecular Plant Pathology. 2008;72(1):10-20; doi: https://doi.org/10.1016/j.pmpp.2008.04.006. 
18. Badri DV, Weir TL, van der Lelie D, Vivanco JM. Rhizosphere chemical dialogues: plant-microbe interactions. Current opinion in biotechnology. 2009;20(6):642-50.

19. Larsbrink J, McKee LS. Bacteroidetes bacteria in the soil: Glycan acquisition, enzyme secretion, and gliding motility. In: Advances in Applied Microbiology. Elsevier; 2020. p. 63-98.

20. Fierer N, Lauber CL, Ramirez KS, Zaneveld J, Bradford MA, Knight R. Comparative metagenomic, phylogenetic and physiological analyses of soil microbial communities across nitrogen gradients. The ISME journal. 2012;6(5):1007-17.

21. Ivanova AA, Zhelezova AD, Chernov TI, Dedysh SN. Linking ecology and systematics of acidobacteria: Distinct habitat preferences of the Acidobacteriia and Blastocatellia in tundra soils. PloS one. 2020;15(3):e0230157.

22. Wang S, Meade A, Lam H-M, Luo H. Evolutionary Timeline and Genomic Plasticity Underlying the Lifestyle Diversity in Rhizobiales. Msystems. 2020;5(4).

23. Sadowsky MJ, Graham PH. Soil biology of the Rhizobiaceae. In: The Rhizobiaceae. Springer; 1998. p. 155-72.

24. Zhou J, Jiang X, Zhou B, Zhao B, Ma M, Guan D, et al. Thirty four years of nitrogen fertilization decreases fungal diversity and alters fungal community composition in black soil in northeast China. Soil Biology Biochemistry 2016;95:135-43.

25. Zhang S, Wang Y, Sun L, Qiu C, Ding Y, Gu H, et al. Organic mulching positively regulates the soil microbial communities and ecosystem functions in tea plantation. BMC microbiology. 2020;20:1-13.

26. Lombard L, Van der Merwe N, Groenewald J, Crous P. Generic concepts in Nectriaceae. Studies in Mycology. 2015;80:189-245.

27. Malapi-Wight M, Salgado-Salazar C, Demers J, Veltri D, Crouch JA. Draft genome sequence of Dactylonectria macrodidyma, a plant-pathogenic fungus in the Nectriaceae. Genome announcements. 2015;3(2).

28. Zhou H, Gao Y, Jia X, Wang M, Ding J, Cheng L, et al. Network analysis reveals the strengthening of microbial interaction in biological soil crust development in the Mu Us Sandy Land, northwestern China. Soil Biology Biochemistry. 2020:107782.

29. Tong A-Z, Liu W, Liu Q, Xia G-Q, Zhu J-Y. Diversity and composition of the Panax ginseng rhizosphere microbiome in various cultivation modesand ages. BMC Microbiology. 2021;21(1):18; doi: 10.1186/s12866-020-02081-2.

30. Zhang S, Sun L, Wang Y, Fan K, Xu Q, Li Y, et al. Cow manure application effectively regulates the soil bacterial community in tea plantation. BMC microbiology. 2020;20(1):1-11.

31. Gundlapally SR, Garcia-Pichel F. The community and phylogenetic diversity of biological soil crusts in the Colorado Plateau studied by molecular fingerprinting and intensive cultivation. Microbial Ecology. 2006;52(2):345-57.

32. Ghoul M, Mitri S. The ecology and evolution of microbial competition. Trends in microbiology. 2016;24(10):833-45. 
33. Gostinčar C, Grube M, De Hoog S, Zalar P, Gunde-Cimerman N. Extremotolerance in fungi: evolution on the edge. FEMS microbiology ecology. 2009;71(1):2-11.

34. Gong L, Gao J, Xu T, Qu J, Wang Z, Yang Z, et al. Transcriptome analysis of field-grown Asian ginseng provides clues to environmental conditions and developmental mechanisms related to red skin root syndrome. Industrial Crops and Products. 2020;153:112486; doi: https://doi.org/10.1016/j.indcrop.2020.112486.

35. Zhao D, Liu B, Li LY, Zhu XF, Wang YY, Wang JQ, et al. Simplicillium chinense: a biological control agent against plant parasitic nematodes. Biocontrol Science and Technology. 2013;23(8):980-6; doi: 10.1080/09583157.2013.809514.

36. Shen J-P, Zhang L-M, Guo J-F, Ray JL, He J-Z. Impact of long-term fertilization practices on the abundance and composition of soil bacterial communities in Northeast China. Applied Soil Ecology. 2010;46(1):119-24.

37. Liu J, Ma Q, Hui X, Ran J, Ma Q, Wang X, et al. Long-term high-P fertilizer input decreased the total bacterial diversity but not phoD-harboring bacteria in wheat rhizosphere soil with available-P deficiency. Soil Biology Biochemistry. 2020:107918.

38. Lambers $\mathrm{H}$, Mougel $\mathrm{C}$, Jaillard B, Hinsinger P. Plant-microbe-soil interactions in the rhizosphere: an evolutionary perspective. Plant soil. 2009;321(1-2):83-115.

39. Hu H, Zhou H, Zhou S, Li Z, Wei C, Yu Y, et al. Fomesafen impacts bacterial communities and enzyme activities in the rhizosphere. Environmental Pollution. 2019;253:302-11.

40. Tian J, Wu B, Chen H, Jiang N, Kang X, Liu X. Patterns and drivers of fungal diversity along an altitudinal gradient on Mount Gongga, China. Journal of Soils Sediments. 2017;17(12):2856-65.

41. Shankar V, Agans R, Paliy O. Advantages of phylogenetic distance based constrained ordination analyses for the examination of microbial communities. Scientific reports. 2017;7(1):1-5.

42. Bian X, Xiao S, Zhao Y, Xu Y, Yang H, Zhang L. Comparative analysis of rhizosphere soil physiochemical characteristics and microbial communities between rusty and healthy ginseng root. Scientific Reports. 2020;10(1):15756; doi: 10.1038/s41598-020-71024-8.

43. Riley D, Barber S. Bicarbonate accumulation and $\mathrm{pH}$ changes at the soybean (Glycine max (L.) Merr.) root-soil interface. Soil Science Society of America Journal. 1969;33(6):905-8.

44. Riley D, Barber S. Salt Accumulation at the Soybean (Glycine Max.(L.) Merr.) Root-Soil Interface. Soil Science Society of America Journal. 1970;34(1):154-5.

45. Murray MG, Thompson WF. Rapid isolation of high molecular weight plant DNA. Nucleic Acids Research. 1980;8(19):4321-5.

46. Zhou J. DNA recovery from soils of diverse composition. Appl Environ Microbiol. 1996;62(2):316-22.

47. Wang Y, Zheng J, Liu X, Yan Q, Hu Y. Short-term impact of fire-deposited charcoal on soil microbial community abundance and composition in a subtropical plantation in China. Geoderma. 2020;359:113992. 
48. Magoč T, Salzberg SL. FLASH: fast length adjustment of short reads to improve genome assemblies. Bioinformatics. 2011;27(21):2957-63.

49. Bokulich NA, Subramanian S, Faith JJ, Gevers D, Gordon JI, Knight R, et al. Quality-filtering vastly improves diversity estimates from Illumina amplicon sequencing. Nature methods. 2013;10(1):57-9.

50. Caporaso JG, Kuczynski J, Stombaugh J, Bittinger K, Bushman FD, Costello EK, et al. QIIME allows analysis of high-throughput community sequencing data. Nature methods. 2010;7(5):335-6.

51. Edgar RC, Haas BJ, Clemente JC, Quince C, Knight R. UCHIME improves sensitivity and speed of chimera detection. Bioinformatics. 2011;27(16):2194-200.

52. Haas BJ, Gevers D, Earl AM, Feldgarden M, Ward DV, Giannoukos G, et al. Chimeric 16S rRNA sequence formation and detection in Sanger and 454-pyrosequenced PCR amplicons. Genome research. 2011;21(3):494-504.

53. Edgar RC. UPARSE: highly accurate OTU sequences from microbial amplicon reads. Nature methods. 2013;10(10):996-8.

54. Quast C, Pruesse E, Yilmaz P, Gerken J, Schweer T, Yarza P, et al. The SILVA ribosomal RNA gene database project: improved data processing and web-based tools. Nucleic acids research. 2012;41(D1):D590-D6.

55. Edgar RC. MUSCLE: multiple sequence alignment with high accuracy and high throughput. Nucleic acids research. 2004;32(5):1792-7.

56. D BS. Soil Agro-chemistrical Analysis. China Agriculture Press; 2000.

57. Algina J, Keselman H. Comparing squared multiple correlation coefficients: Examination of a confidence interval and a test significance. Psychological methods. 1999;4(1):76.

58. Legendre P, Anderson MJ. Distance-based redundancy analysis: testing multispecies responses in multifactorial ecological experiments. Ecological monographs. 1999;69(1):1-24.

\section{Supplementary Information}

Table S1. Statistical results of bacterial sequencing data processing.

Table S2. Statistical results of fungal sequencing data processing.

Table S3. Network topological characteristics of microbial communities.

Table S4 Fungus in interaction networks that may be associated with diseases.

Figure S1. The rarefaction curves of observed OTUs at OTU level across all samples.

Figure S2. MetaStat analysis of bacterial abundance from phylum level to species level.

Figure S3. MetaStat analysis of fungi abundance from phylum level to species level.

Figure S4. PH of rhizosphere soil. 
Figure S5. Nutrients of rhizosphere soil.

Figure S6. Enzyme activities of rhizosphere soil.

\section{Tables}

Table 1 Diversity of the 16S rRNA gene-based bacterial and ITS rRNA gene-based fungi communities. Values are the means \pm standard errors $(n=6)$. Different letters in the same row mean significant difference at $P<0.05$ among the five treatments. HG (healthy ginseng), GRS1 (rust root area 0 to $25 \%$ ), GRS2 (rust root area 25 to $50 \%$ ), GRS3 (rust root area 50 to $75 \%$ ) and GRS4 (rust root area 75 to $100 \%$ ).

\begin{tabular}{|c|c|c|c|c|c|}
\hline \multicolumn{6}{|c|}{ Bacteria } \\
\hline & OTUs & $\begin{array}{l}\text { Observed } \\
\text { species }\end{array}$ & Shannon & Simpson & Chao1 \\
\hline$H G$ & $2209 \pm 163 b$ & $1952 \pm 125 b$ & $\begin{array}{l}8.518 \pm 0.078 \\
\text { bc }\end{array}$ & $0.993 \pm 0.001 b$ & $\begin{array}{l}2171.36 \pm 140.73 \\
\text { bc }\end{array}$ \\
\hline GRS1 & $2226 \pm 183 b$ & $2013 \pm 111 a b$ & $\begin{array}{l}8.604 \pm 0.116 \\
\text { bc }\end{array}$ & $0.993 \pm 0.001 \mathrm{~b}$ & $\begin{array}{l}2400.47 \pm 365.58 \\
a b\end{array}$ \\
\hline GRS2 & $2510 \pm 198$ a & $2225 \pm 156$ a & $\begin{array}{l}8.849 \pm 0.073 \\
a\end{array}$ & $0.994 \pm 0.000 \mathrm{a}$ & $2475.05 \pm 166.26 \mathrm{a}$ \\
\hline GRS3 & $2124 \pm 191 b$ & $1874 \pm 168 b$ & $8.369 \pm 0.219 c$ & $0.991 \pm 0.002 b$ & $2096.48 \pm 182.25 \mathrm{c}$ \\
\hline GRS4 & $\begin{array}{l}2297 \pm 250 \\
\mathrm{ab}\end{array}$ & $2035 \pm 219 a b$ & $\begin{array}{l}8.606 \pm 0.254 \\
b\end{array}$ & $0.992 \pm 0.002 b$ & $\begin{array}{l}2278.28 \pm 245.57 \\
\text { abc }\end{array}$ \\
\hline \multicolumn{6}{|l|}{ Fungi } \\
\hline & OTUs & $\begin{array}{l}\text { Observed } \\
\text { species }\end{array}$ & Shannon & Simpson & Chao1 \\
\hline $\mathrm{HG}$ & $593 \pm 103 a$ & $533 \pm 85 a b$ & $\begin{array}{l}4.822 \pm 0.111 \\
\mathrm{a}\end{array}$ & $0.913 \pm 0.005 a$ & $576.98 \pm 102.37 \mathrm{ab}$ \\
\hline GRS1 & $602 \pm 132 a$ & $537 \pm 114 a b$ & $\begin{array}{l}4.866 \pm 0.243 \\
a\end{array}$ & $\begin{array}{l}0.927 \pm 0.009 \\
a b\end{array}$ & $595.66 \pm 134.21 a b$ \\
\hline GRS2 & $615 \pm 84 a$ & $568 \pm 84 a$ & $4.74 \pm 0.209 \mathrm{a}$ & $\begin{array}{l}0.914 \pm 0.015 \\
a b\end{array}$ & $717.49 \pm 244.41 \mathrm{a}$ \\
\hline GRS3 & $489 \pm 93$ a & $436 \pm 75 b$ & $4.348 \pm 0.0 .5 b$ & $0.905 \pm 0.008 b$ & $486.28 \pm 86.68 b$ \\
\hline GRS4 & $546 \pm 140$ a & $489 \pm 120 \mathrm{ab}$ & $\begin{array}{l}4.497 \pm 0.263 \\
b\end{array}$ & $0.897 \pm 0.016 b$ & $549.05 \pm 139.95 \mathrm{ab}$ \\
\hline
\end{tabular}



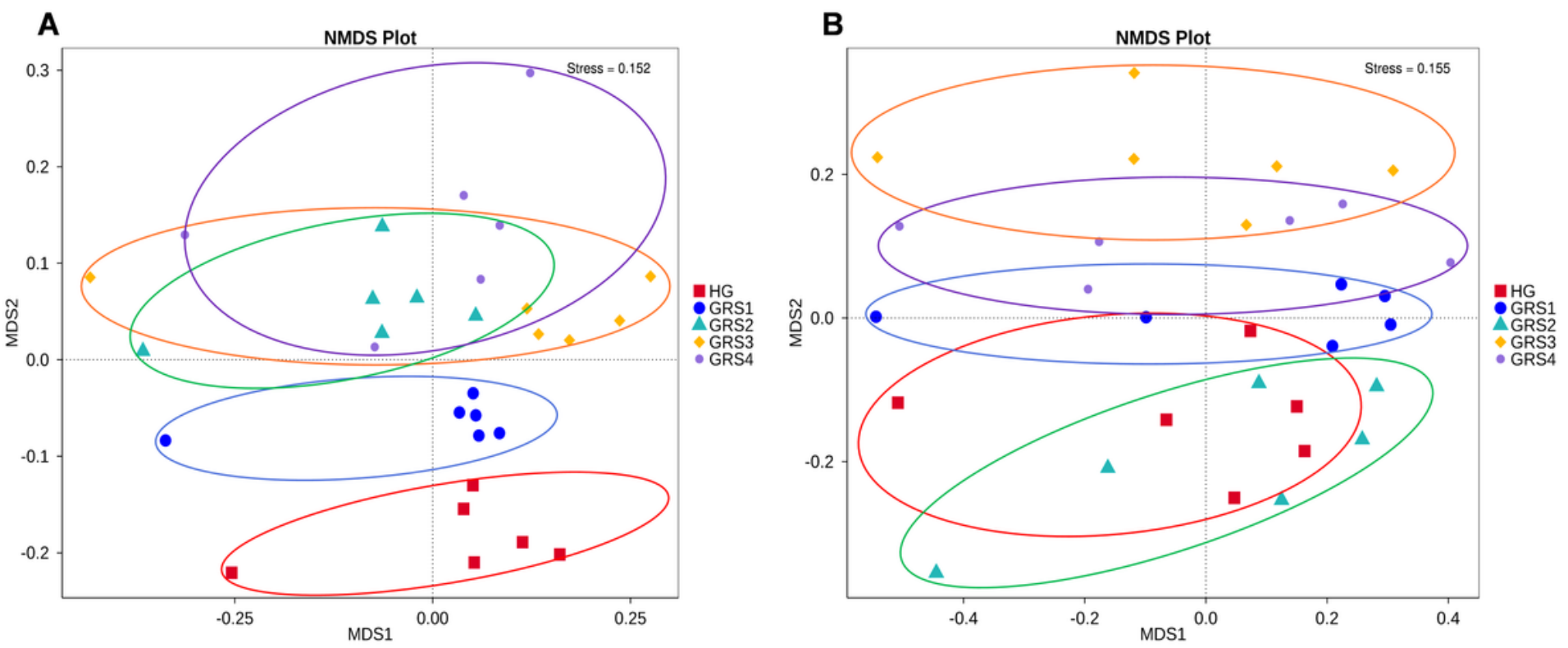

Figure 1

Non-Metric Multi-Dimensional Scaling (NMDS) analysis plot. A) bacteria; B) fungi. HG (healthy ginseng), GRS1 (red skin root area 0 to 25\%), GRS2 (red skin root area 25 to 50\%), GRS3 (red skin root area 50 to $75 \%$ ) and GRS4 (red skin root area 75 to $100 \%$ ).
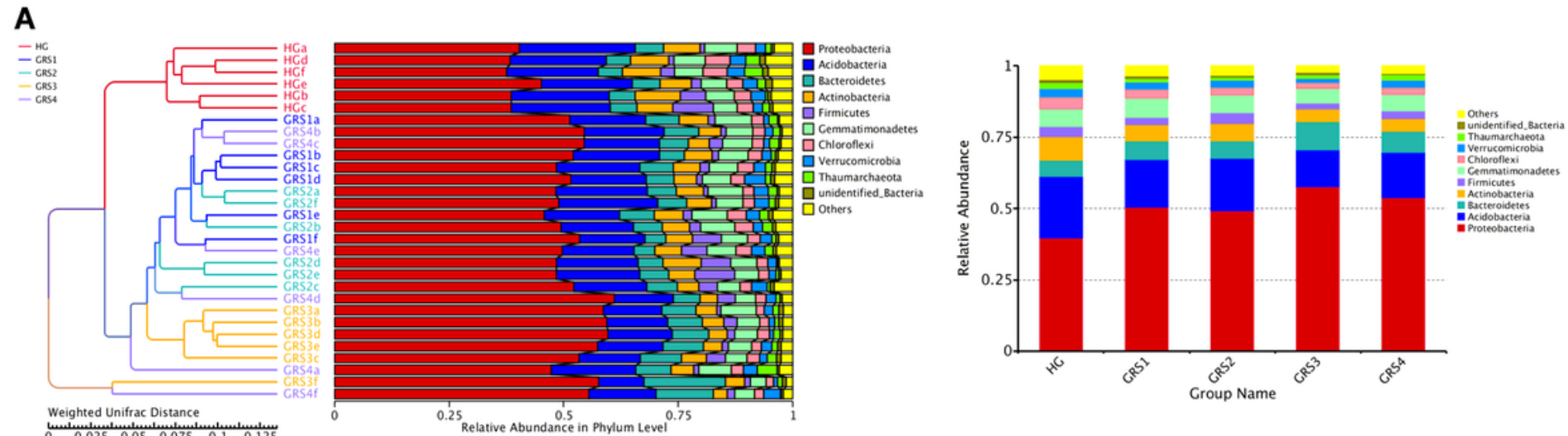

B

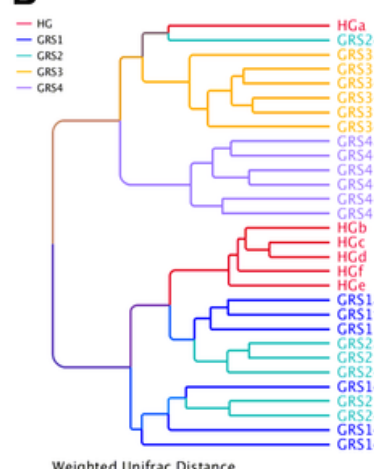

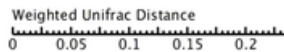
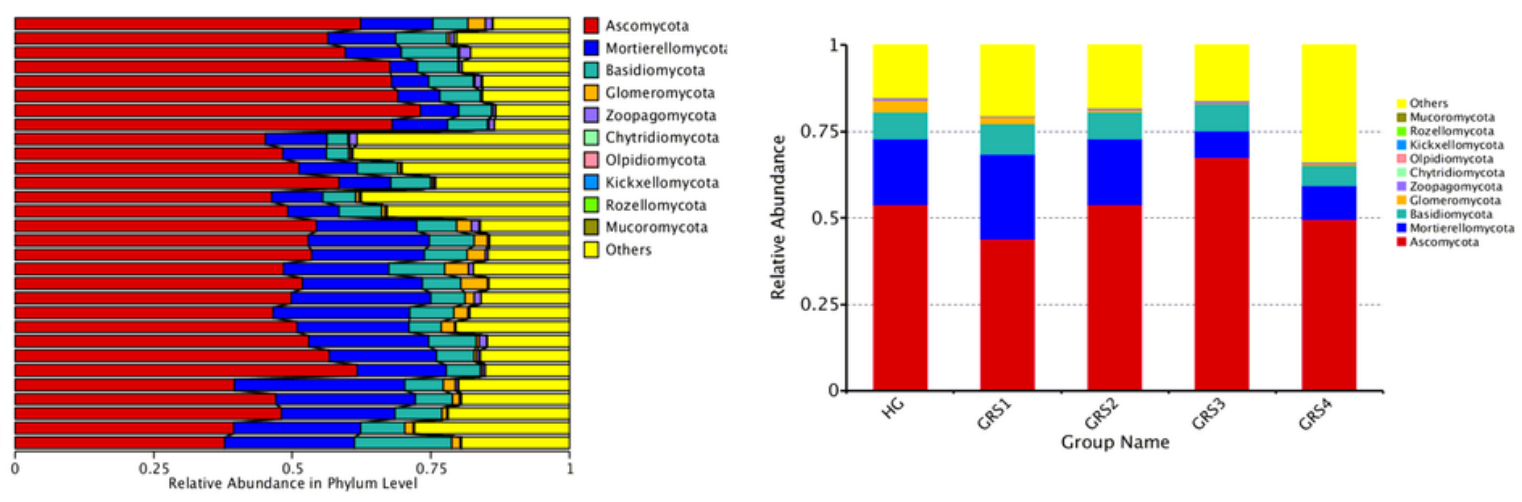

Figure 2 
Unweighted Pair-group Method with Arithmetic Mean (UPGMA) clustering analysis with Weighted Unifrac distance matrix and the relative abundance of each sample and group at the phylum level. A) bacteria; B) fungi.
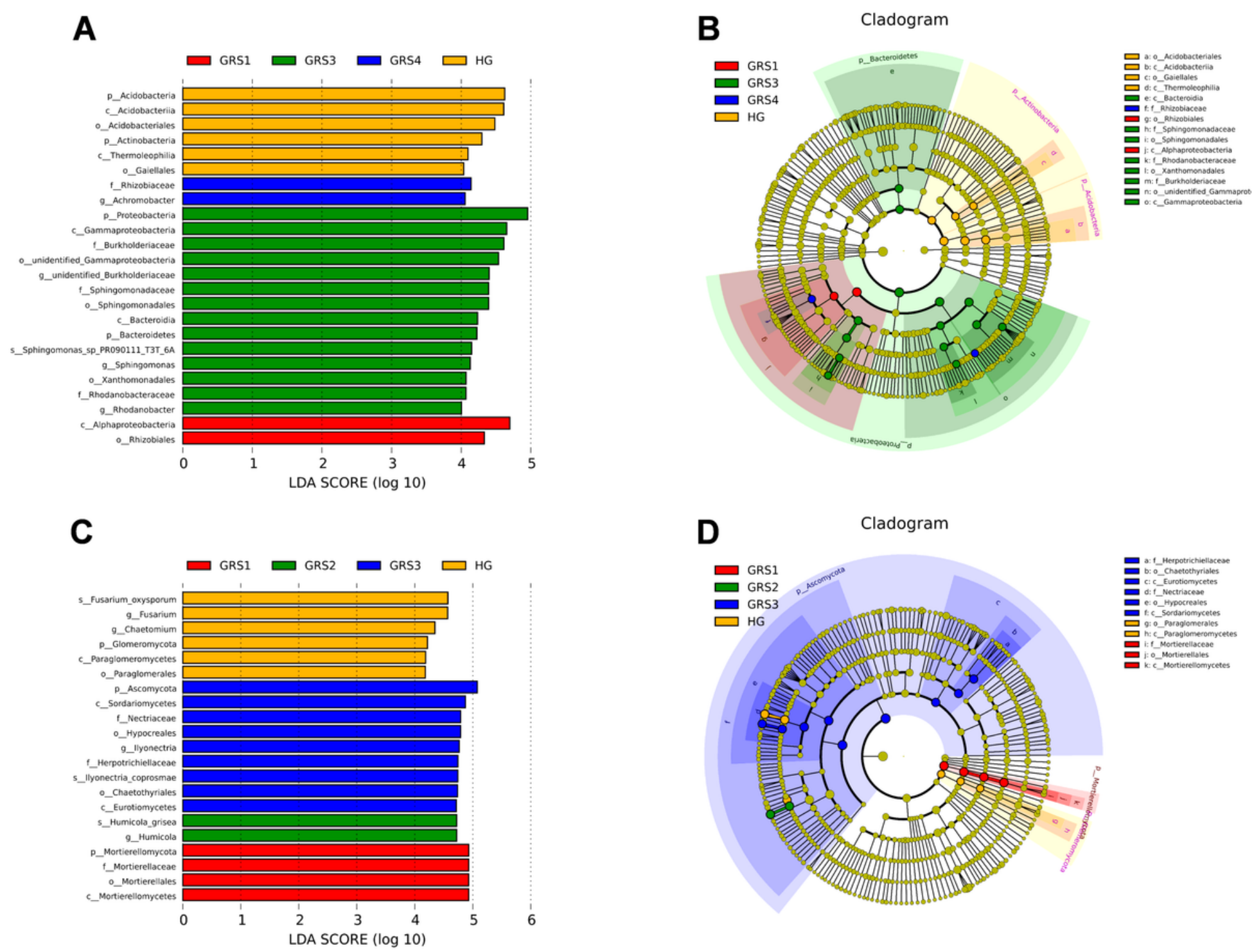

\section{Figure 3}

The linear discriminant analysis (LDA) effect size (LEfSe) analysis. A) LDA scores of bacteria differential taxa (LDA score>4); B) diagram of bacterial differential taxa; C) LDA scores of fungi differential taxa (LDA score $>4$ ); D) diagram of fungi differential taxa. 


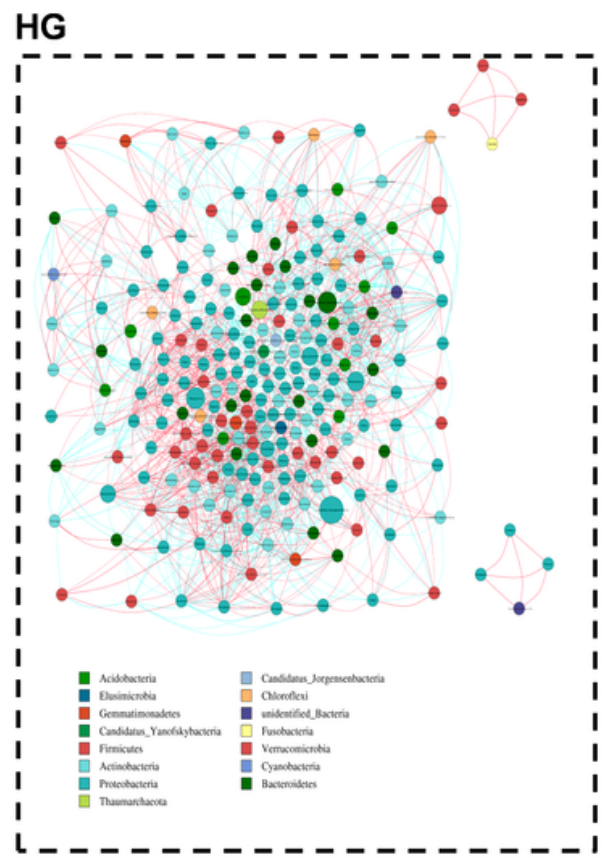

\section{GRS3}

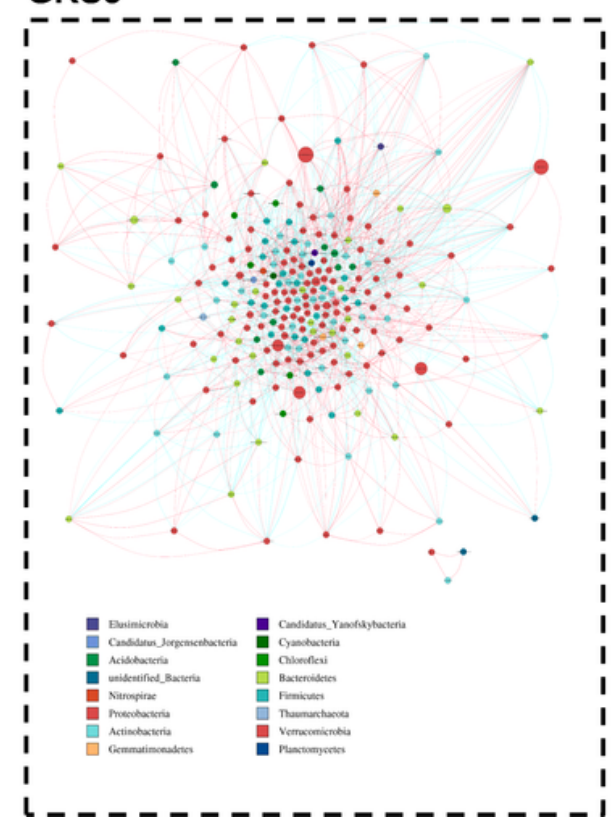

\section{GRS1}

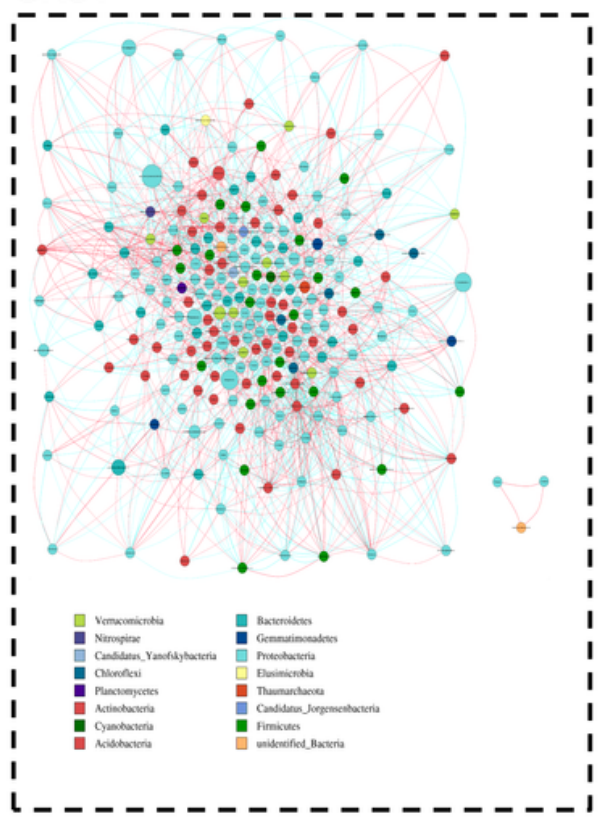

GRS4

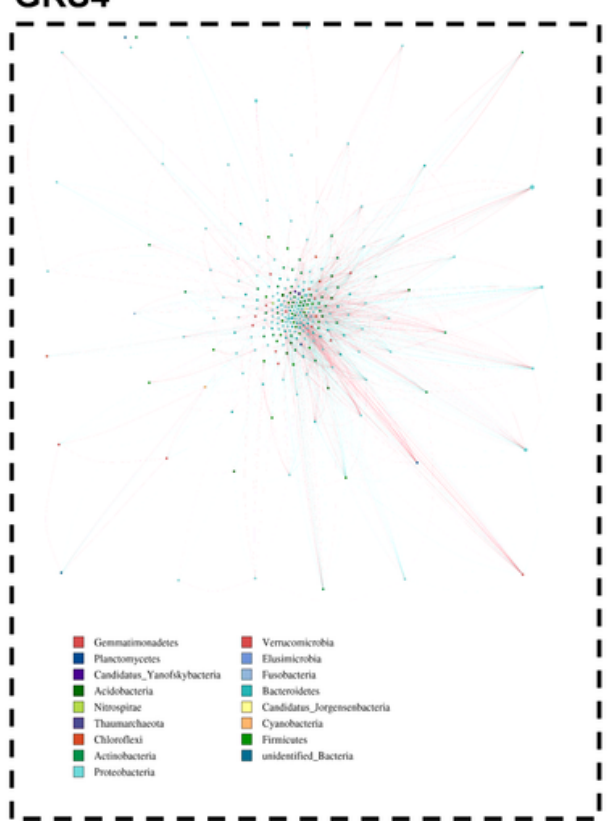

Figure 4

Overview of bacterial networks. Different nodes represent different genera; the size of nodes represents the average relative abundance of the genus; the nodes of the same gate have the same color; the color of the lines between nodes corresponds to the positive and negative correlation (red is positively correlated; blue is negatively correlated). 

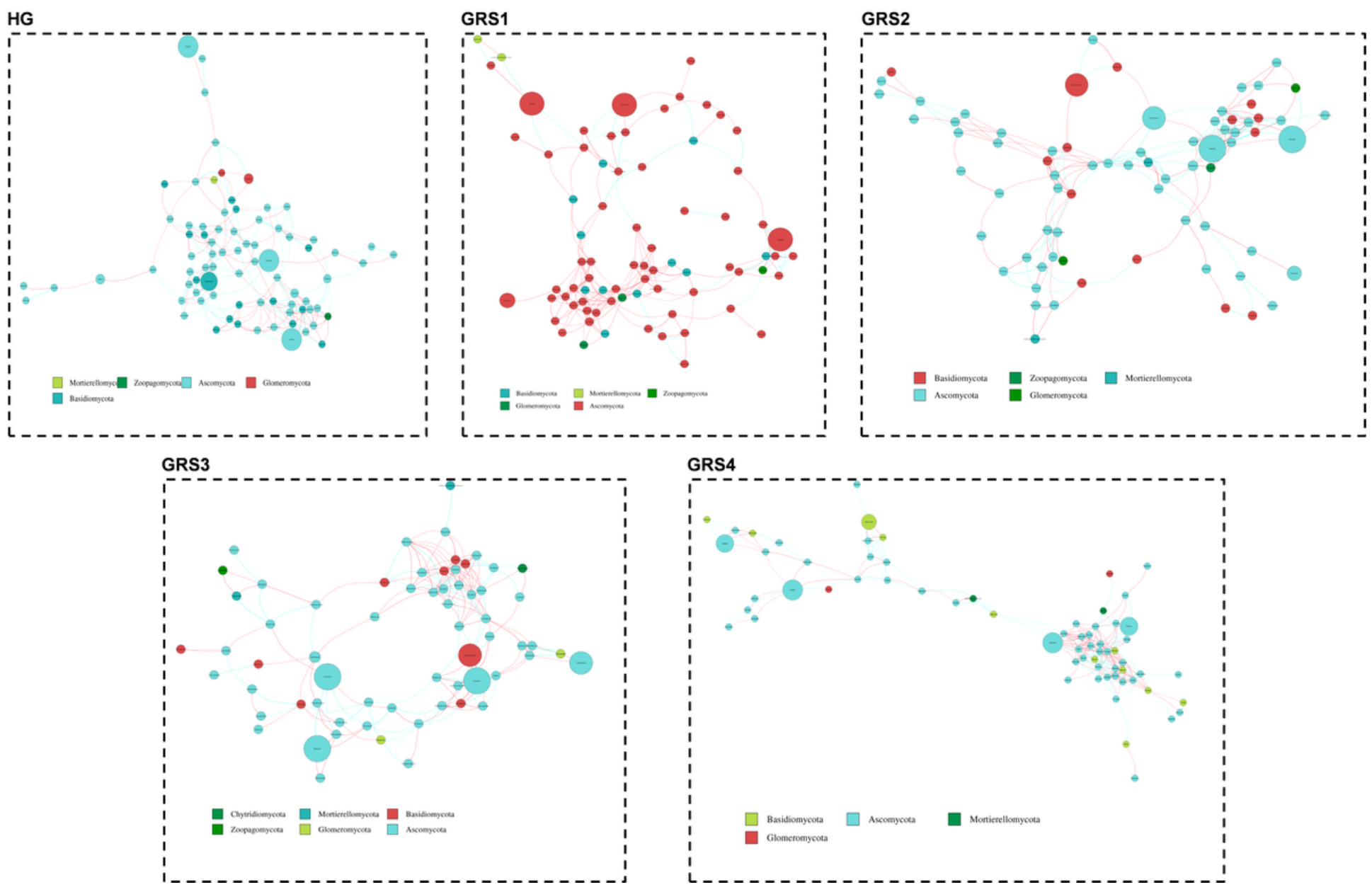

\section{Figure 5}

Overview of fungi networks. Different nodes represent different genera; the size of nodes represents the average relative abundance of the genus; the nodes of the same gate have the same color; the color of the lines between nodes corresponds to the positive and negative correlation (red is positively correlated; blue is negatively correlated).
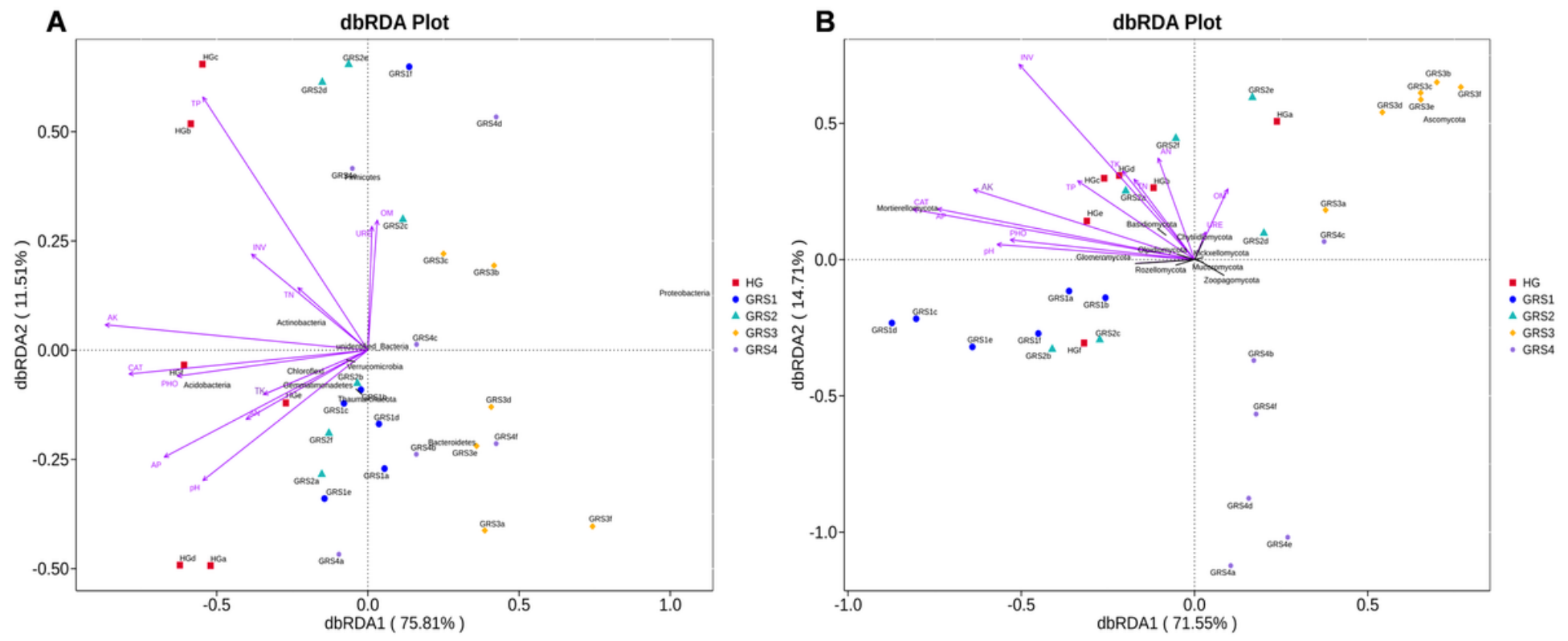
Figure 6

Distance-based redundancy analysis (dbRDA) at the phylum level. A) bacteria; B) fungi. The length of arrows represents the degree of correlation between environmental factors and community distribution and species distribution.

\section{Supplementary Files}

This is a list of supplementary files associated with this preprint. Click to download.

- Supplementarymaterial.docx 